\title{
Negative Transfer of Mother Tongue in Second Language Acquisition and Related Teaching Strategies
}

\author{
Lu Liu \\ School of Foreign Studies, Xi'an City, Shaanxi Province, 710065, China
}

Keyworks: Second Language Acquisition; Negative Transfer; Mother Tongue; Teaching Strategies

\begin{abstract}
Language transfer is an important factor that influences language learning in second language acquisition. This paper discusses and analyzes the language transfer from phonetics, vocabulary, syntax and pragmatics perspectives during second language acquisitions, and then puts forward some teaching strategies to reduce their impacts and improve the language learning effects.
\end{abstract}

\section{Introduction}

Transfer is a psychological term, which means an influence of the existing knowledge and skills of a learner on the new knowledge or skills acquisition during a learning process. Language transfer means that language learners unconsciously adopt rules of mother tongue to deal with target language information due to unfamiliarity with rules of target languages when learning second language or foreign language. Language transfer could be divided into two aspects, respectively are the positive transfer and the negative transfer. When the mother tongue is similar with the target language, the positive transfer is often conducted, contributing to the acquisition of the second language. When there is a big difference between the mother tongue and the second language, language learners refer too much to certain rules of the mother tongue, so that the negative transfer occurs which establishes obstacles and disturbances to the second language acquisition. However, Chinese and English belong to different language families, so more energy should be placed on the negative transfer in order to arouse the attention of teachers and students, and minimize its effects during second language learning.

\section{Negative Transfer of Mother Tongue in Second Language Acquisition}

Negative transfer of phonetics. It is obvious that no matter how fluent Chinese people speak English, they more or less take a bit of Chinese accent and cannot be pure like native speakers. The main reason is that once learners have missed the critical period of language learning, a foreign language learning can not be as relaxed and natural as a mother tongue learning, so that phonetics must be negatively affected by the pronunciation and rules of mother tongue. From the perspective of English pronunciation, English is a kind of intonation language which mainly relies on intonation to distinguish its semantics, but Chinese belongs to a tone language that distinguishes the semantics through its tones. Therefore, both of above two languages differ in the number and combination of phonemes greatly. Some English phonemes are not existed in Chinese, which make Chinese students tend to find similar sounds in pinyin (familiar phonemes they prefer). Furthermore, English has both an open vowel ending and a closed syllable ending, while Chinese usually ends with a vowel. Therefore, Chinese students often add a vowel at the end of English Consonants

Negative transfer of vocabulary. Because of different historical backgrounds, the vocabulary of English and Chinese contains rich cultural connotations, and a lot of words which seem to be equal have different meanings. For example, the cultural connotation of "intellectual" and "rest room" is not equal to their conceptual meanings, as well as the concept of equivalents in Chinese, so that a direct copying is bound to make mistakes. Then, in a sentence that "Fresh air will do good to your body", the misuse of body is affected by the negative transfer of mother tongue, because English learners equate the word "body" with the body in Chinese. In Chinese, the "body" has two 
meanings, respectively are body and health. It is obvious that the "body" could be altered by the "health" in this sentence.

Negative transfer of syntax. There are also differences in syntax between English and Chinese. Chinese emphasizes parataxis, while English emphasizes hypotaxis. The word order of English is mostly $\mathrm{S}+\mathrm{V}+\mathrm{O}$, but the word order of Chinese largely depends on the meaning of sentence. Therefore, compared with Enligh sentence, the order of subject and verb is more flexible in Chinese sentences. In Chinese, some adverbs of frequency, such as "always" and "usually", are comparatively fixed, often in front of verbs. In English, its word order depends on the type of predicate verbs. Since there are differences in word order between English and Chinese, foreign language learners often suffer from misuse of word orders. For example, "He often is the first to come". In Chinese, there is often a phenomenon of omission of clausal subjects, which leads to errors in subject vacancy or verb dangling when transferred to English. Chinese students are prone to make following mistakes in their writing, such as "As a small child, her parents often took her to the countryside". However, in the sentence that "As a small child”, the logical subject should be "she", so that the main subject should be consistent with this. Therefore, the correct translation is that "As a small child, she was often taken to the countryside.".

Negative transfer of pragmatics. The negative transfer of pragmatics means the existing pragmatics has disturbing effects on the acquisition of second languages and intercultural communications. Generally speaking, compared with phonetics and syntax, the two sides of communication cannot tolerate the pragmatic failure in communication. Therefore, the negative transfer of pragmatics is regarded as the most noteworthy aspect of English learning and communication. For example, due to the influence of negative transfer of pragmatics, a Chinese student is likely to show friendliness to a foreign friend who is about to leave by saying "Where are you going ?" or "What are you going to do ?". In general, in China these greetings do not expect full answers, or the misunderstanding or displeasure cannot appeared when listeners' answer is not transparent enough. However, foreign friends may feel a little awkward and answer truthfully "I just want to go to the tailor". Therefore, it can be said that due to pragmatic failures, Chinese greetings in this case did not achieve desired effect.

\section{Strategies in Foreign Language Teaching}

The contrastive analysis. During the second language acquisition, teachers could compare the phonetic system of English and Chinese by the contrastive analysis, and reduce confused phonemes and their pronunciations. In addition, difficulties in pronunciations are stressed to guide students to pay more attention on them. Then the pronunciation mistakes which Chinese students often make are listed, and the possible pronunciation difficulties are predicted so as to point out and correct effectively. At last, enhancing students' interests in phonetics learning through readings, original movie imitations, speech contests and etc..

Broad learning around words. The vocabulary learning in second language acquisitions is not only a one-time learning of words, but a series of broad meanings around a word. To different meanings of words, putting them in a whole sentence and forming the deepest processing of the brain so as to ensure the best memory effect. In general, vocabulary learning always conducted through indirect means, such as the reading, writing, conversation, communication and etc.. Practice shows that extensive reading is an effective way of vocabulary learning. Discourses provide specific contexts and collocation habits of a vocabulary learning, which effectively reduces the negative transfer of vocabulary resulting from the ignorance of the cultural connotations of the target language. However, speaking exercises could increase students' productive vocabulary. In school, the spoken English corner, various forms of English speaking contests and recitation contests are good opportunities for a oral practice. At last, writing not only requires mastery and application of productive vocabulary, but also involves a series of more complex problems, such as the accuracy of words, the ingenuity of sentence, the natural cohesion of words and so on

Reducing the negative transfer of syntax. The language input to students should be increased to reduce the negative transfer of syntax. The Input Hypothesis is the core section of Krashen 
second language learning theory. He considered the most optimum input should have the following features. Firstly, intelligibility, which means input information should be slightly higher than the existing language proficiency of learners. Secondly, interesting \& relevant. the more interesting input languages are, the more relevant they are, and the weaker emotional filters are, the more materials are absorbed by learners. Thirdly, enough input, meaning that a few exercises and essays are not enough to acquire a new language structure, and the amount of input should be enough and their forms could be varied. Teacher oriented language input does not enable students to understand and use what they have learned in the true sense. Therefore, it is essential to motivate students' interests to learn English.The popularization of modern teaching equipment provides teachers with various ways of language input. Furthermore, teachers arrange for students to perform English skits with certain difficulty, so that students could organize their own language, and take the initiative to surf the Internet, collect relevant information and arouse their enthusiasm for learning English.

Culture learning during second language acquisition. At last, second language acquisition is not only a learning of linguistic structures of target language, a learning of phonetics and text symbol systems, as well as the customs, religious beliefs, science and technology, literature, art and other dominant forms of culture in target nations. In foreign language teaching, teachers should consciously expand the students' understanding of national culture and actively enlighten the students in the process of information processing, then the cultural information in the target language is constantly stored in the memory of the brain. Teachers can also set up some cultural atmosphere and scenes, such as shopping, booking rooms, complaints, asking for directions and so on, to allow students imitate real life and make situation dialogues to improve students' awareness of cultural differences.

\section{Summary}

For students who study English in a Chinese environment, the amount of Chinese has been quite rich in their brain, so that it is inevitable that Chinese interferes with English when learning a second language information. Based on this situation, teachers only provide enough and standardized English input for students, guide students to learn more about British and American culture and treat their inter-language bias scientifically, in which way the negative transfer could be effectively overcome and achieve an excellent teaching effect.

\section{References}

[1] Man Guo. The Influence of Negative Transfer of Mother Tongue on Japanese Writing and Its Countermeasures [D]. Guangxi University, 2015.

[2] Yuanyuan Chai. Negative Transfer of Mother Tongue in Second Language Acquisition and Its Teaching Strategies [J]. 2014, (02): 115-118.

[3] Li Wang. Negative Transfer of Mother Tongue in English Writing [D]. Harbin Institute of Technology, 2013.

[4] Tingting Zhu, Qiuwei Zhao, Li Sun. Negative Transfer of Mother Tongue in Second Language Acquisition and Its Strategies - Taking Japanese Vocabulary Learning as examples. Journal of Jiamusi Normal Universiy, 2012, (11): 373.

[5] Lizhong Niu. A Study of Negative Transfer of Mother Tongue in Two Language Acquisition - a Case Study of Chinese Speaking Japanese Learners [J]. Journal of Tonghua Normal Universiy. 2012, (03): 54-57.

[6] Ying Wu. Negative Transfer of Mother Tongue in SecondLanguage Acquisition and Measures. Chongqing University of Science \& Technology (Social Sciences Edition), 2010, (2): 188-189.

[J]. Liping Duan. A Cognitive Approach to the Phenomenon of Language Transfer in Translation Learning [D]. Chongqing University, 2008. 